\title{
Analysis of arterial flowmotion in spinal cord injured and elderly subjects in an area at risk for the development of pressure sores
}

\author{
V Schubert ${ }^{1}, \mathrm{P}-\AA$ Schubert $^{2}, \mathrm{G} \mathrm{Breit}^{3}, \mathrm{M}$ Intaglietta $^{4}$ \\ ${ }^{1}$ Department of Clinical Neuroscience and Family Medicine, Division of Geriatric Medicine, Karolinska Institutet, \\ Huddinge University Hospital, S-141 86 Huddinge; ${ }^{2}$ Department of Electronics, Royal Institute of Technology, \\ Stockholm, Sweden; ${ }^{3}$ Life Science Division, NASA-Ames Research Center, Moffett Field; ${ }^{4}$ Department of \\ Applied Mechanics and Engineering Sciences, University of California-San Diego, La Jolla, USA
}

\begin{abstract}
Flowmotion (blood flow changes due to vasomotion) in the skin over a risk area for pressure sores, the sacrum, and a non-risk area, the gluteus muscle, was evaluated by laser Doppler fluxmetry during resting conditions and post-occlusive reactive hyperaemia (PRH) response. Measurements were made in healthy younger subjects and in two risk groups for pressure sores-spinal cord injured (SCI) and the elderly. The SCI were divided into two subgroups, one with and one without distinct flowmotion seen on the original recordings over the sacrum. The Prony spectral line estimation (PSLE) method was used to determine the power spectrum of the flowmotion activity. During the PRH, flowmotion frequencies were found in two separated bands, 5.4-6.6 cpm (cycles $\min ^{-1}$ ) and 7.8-9.0 cpm. In the subgroup without distinct flowmotion, the PSLE method found flowmotion frequencies similar to the other groups. During the PRH, the flowmotion power pattern over the sacrum was similar within all groups, but the power was extremely low in one subgroup of SCI subjects. To conclude, flowmotion (vasomotion) was present in the two skin areas and increased during the PRH response, reaching a maximum within $50 \mathrm{~s}$. This study shows that the flowmotion frequencies might be locally driven, whereas the power might be centrally mediated. Disturbances in microcirculatory flowmotion can be a part of the mechanisms leading to skin ischaemia and pressure sores. A new method has been developed for analysing differences in flowmotion behaviour such that statistical comparisons can be made.
\end{abstract}

Keywords: aged; decubitus ulcer; laser Doppler fluxmetry; Prony spectral line estimation; spinal cord injured; vasomotion

\section{Introduction}

Tissue breakdown in the skin, leading to pressure sore formation, is a common complication among patients with spinal cord injuries, especially during their immediate post-injury period, ${ }^{1}$ and among elderly patients in close relation to a hip fracture. ${ }^{2}$ A deep pressure sore will interfere with the patient's quality of life, affect the rehabilitation, and increase the cost for wound healing.

Pressure sore formation is a multifactorial process which has recently been addressed in a new way, by evaluation of the skin microcirculation under various conditions from one of the most susceptible areas, the sacrum area..$^{3-7}$. The microcirculation in the sacrum area in these two well-known risk groups showed impairment in the transitory increase of cutaneous blood flow, the so-called reactive hyperaemia response. Reactive hyperaemia response is an indication of recovery to meet the demands for repair which follow a period of arterial occlusion. Impaired reactive hyperaemia in SCI patients occurs when there is neuronal damage, in which case it is often accompanied by a high resting blood flow. ${ }^{3}$ In elderly patients the impairment could be due to either a relatively decreased microvascular density that is known to occur with age, or to a reduced blood flow in the skin microcirculation. ${ }^{4}$

Rhythmic variations of the microvascular flow, identified as flowmotion (blood flow changes due to vasomotion), have been registered from the sacral area both during resting conditions and during the decreasing phase of the post-occlusive reactive hyperaemia response. ${ }^{3,5}$ Vasomotion is defined as spontaneous rhythmic variations in the diameter of the small vessels, caused by contractions and relaxations of the muscular components of their walls. Its activity is centrally mediated through the nervous system and circulating hormones, but is also locally controlled by myogenic mechanisms. ${ }^{8,9}$ In terminal arterioles vasomotion has a local origin, ${ }^{10}$ and it is postulated to be due to a vascular pacemaker-like mechanism ${ }^{11}$ that can be influenced by the perfusion pressure, causing the 
arterioles to constrict and dilate spontaneously. Secomb et $a^{12}$ have drawn the important conclusion, that the essential function of vasomotion must be to modulate mass transport in ways that are not possible under static conditions. Thus, vasomotion is believed to be a normal phenomenon in small arteries and arterioles, ${ }^{13}$ and it is an important mechanism for regulating the blood flow in the terminal arterioles and the subsequent downstream capillaries, supplying adequate amounts of oxygen and nutrients to ischaemic areas, washing away waste products from vascular tissues, and it serves as a positive influence in maintaining an effective venous outflow as well. It has been shown, ${ }^{14}$ that the average value of flow in a vessel is highly dependent on the detailed shape of the time variation of the vessel diameter.

Most reports on cutaneous flowmotion are based on measurements using the laser Doppler technique, ${ }^{15}$ but to our knowledge no analyses of the different frequency and power components of flowmotion in a high risk area for pressure sore have previously been made.

The aim of the present investigation was to evaluate the effects of locally applied skin pressure on the flowmotion of the skin microcirculation, in both a high risk and a non-risk skin area for pressure sores, and to characterize the spectral composition of the flowmotion patterns using the Prony spectral line estimation (PSLE) method.

\section{Methods}

\section{Subjects}

Fifty subjects were studied. All SCI and most of the elderly subjects had previously had pressure sores, but none had any sign of tissue damage or ulcer in the areas studied at the time of the investigation. Arterial blood pressure was measured and mean blood pressure $(\mathrm{mBP})$ is defined as the diastolic blood pressure plus one-third of the pulse pressure. The subjects were divided into the following groups.

Group 1 (G1) comprised 10 healthy subjects with a mean age of 36 years (range 22-54), and a blood pressure (BP) of $128 / 70 \pm 4 / 2 \mathrm{~mm} \mathrm{Hg}$ with a mBP of $90 \pm 2 \mathrm{~mm} \mathrm{Hg}$.

Group 2 (G2) comprised 20 elderly subjects with a mean age of 80 years (range 67-97), and a BP of $150 / 84 \pm 5 / 2 \mathrm{~mm} \mathrm{Hg}$ with a $\mathrm{mBP}$ of $106 \pm 3 \mathrm{~mm} \mathrm{Hg}$. This group comprised 10 elderly subjects living at home (mean age 74 years, range 67-84), and 10 patients permanently institutionalized on a geriatric ward (mean age 85 years, range $74-97$ ).

Group 3 (G3) comprised 20 subjects with a spinal cord injury (SCI), a mean age of 38 years (range 21-53), and a BP of $118 / 73 \pm 3 / 2 \mathrm{~mm} \mathrm{Hg}$ with a $\mathrm{mBP}$ of $88 \pm 2 \mathrm{~mm} \mathrm{Hg}$. This group comprised 10 tetraplegic subjects (mean age 38 years, range 21-53) with spinal cord injury at the $\mathrm{C} 2-7$ level, and 10 paraplegic subjects (mean age 39 years, range 23-53) with spinal cord injury at the T5-12 level. All had been injured for at least 1 year.
Design

The local skin microcirculation was characterized using the laser Doppler fluxmeter (Periflux ${ }^{\circledR}$ PFlb, Perimed, Stockholm, Sweden). The measurements were performed at a bandwidth of $12 \mathrm{kHz}$, a gain setting of $\times 10$, and a time constant of $0.2 \mathrm{~s}$. These settings were found to be optimal for recording the post-occlusive reactive hyperaemia response. The laser Doppler fluxmeter was connected to a pen recorder using a paper speed of $120 \mathrm{~mm} \mathrm{~min}^{-1}$. The measurements were made over the sacrum, in an area $10-12 \mathrm{~cm}$ below a line between the iliac crests. The reference point over the gluteus maximus muscle was situated on a line from the sacrum to the greater trochanter in an area $10-12 \mathrm{~cm}$ from the midpoint of the sacrum.

An external pressure was applied to the skin with a specially designed device which has been previously described. ${ }^{6}$ This device consisted of a pivoted arm equipped with a pressure cup on its front end. The pressure cup was made of plexiglass and had a loose bottom disc which could be fastened to the skin with double-sided adhesive tape. The laser Doppler probe was placed in a probe holder fastened to the center of the bottom disc. In order to study the PRH response, the pressure cup was lifted from the skin, leaving the bottom disc and the laser Doppler probe attached to the skin. ${ }^{4}$

The patients were made comfortable in the prone position, while the laser Doppler output was observed for artefacts and transients which usually disappeared after an initial period of approximately $10 \mathrm{~min}$. At this time a control record of the SBF at rest was taken, lasting $3 \mathrm{~min}$. Arterial occlusion was then performed for $3 \mathrm{~min}$ with an external skin pressure of $400 \mathrm{~mm} \mathrm{Hg}$ $(53.3 \mathrm{kPa})$. During the arterial occlusion the laser Doppler signal fell to a level slightly above the zero value of the instrument, the so-called biological zero value. ${ }^{16}$ There is no need to take this value into account in analyses of flowmotion activity. When the pressure was released the laser Doppler signal increased, reached a peak value, and then decreased. The PRH was recorded during $9 \mathrm{~min}$ after pressure release. Rhythmic variations of the laser Doppler signal (flowmotion) were registered both during resting conditions and the PRH period. The mean room temperature was $23.2^{\circ} \mathrm{C}$ (range $20.9-24.4^{\circ} \mathrm{C}$ ).

\section{Data analysis}

The numerical evaluation of the spectral content in the flowmotion patterns on the relatively short original SBF recordings at hand has been based on the so-called Prony spectral line estimation (PSLE) method. ${ }^{17}$ The main advantage of the PSLE method is its ability to analyze short data records, in contrast to the fast Fourier transform (FFT), as has been shown by Meyer and Intaglietta. ${ }^{18}$ Due to its good capability for frequency resolution, it is especially well suited for analysis of data sequences related to vasomotion, that contain a finite number of local oscillators.

In order to study how the flowmotion characteristics 
changed during the course of time, the time axis was divided into three adjacent, non-overlapping intervals (Figure 1, time windows: T1, T2, T3), each $25 \mathrm{~s}$ long, starting from the point of the peak SBF. The original recordings of the PRH response were accordingly split into three segments, one for each time window. The recordings of the resting conditions (RF) were also analyzed using a time window of $25 \mathrm{~s}$.

In order to make statistical comparisons possible between different groups of subjects, some essential attributes have to be extracted from the outcome of the PSLE evaluation. Here, only a short qualitative description is done. Two characteristic features are defined for the flowmotion pattern: (1) a measure of its strength, named the power; and (2) a measure of its rapidity of variation, named the center frequency. Both these features are first computed for each individual SBF record segment, and thereafter group mean values of these features are computed. Subsequently, traditional statistical methods for comparison of arithmetic mean values can be used. Further, the PSLE method implies that the power is built up by contributions from several frequencies. The relative strength of these contributions is presented in the form of a so-called power spectrum. A quantitative mathematical description of these concepts is presented in Appendix 1.

Others have previously evaluated flowmotion frequencies manually, by counting the number of relative maxima per minute. For the purpose of comparison, the frequency with the dominant amplitude of each record has been manually evaluated in this study, partly for the period of resting conditions and partly for the PRH period ( $75 \mathrm{~s})$. The mean value obtained this way, named the dominant frequency for the time period studied, has been compared with the center frequency determined from the PSLE analysis. In this comparison the arithmetic average of the center frequencies for the three time windows (T1-T3) of the PRH period was used.

Group data for frequency and power are presented as mean values \pm standard error of the mean (SE) in the tables, and with error bars (SE) in Figure 2. Normality tests were performed which showed that the data, in relevant cases after performing a square root transformation, did not show any significant deviations from normal distributions. The group data were then compared using the paired or unpaired Student's $t$ test. A $P$ value of 0.05 or less was considered significant.

A test for reproducibility of the skin flowmotion was made in one subject from G1 on five separate occasions during a period of 2 weeks. Measurements were made over both the sacrum and the gluteus regions. On all occasions, rhythmic variations of the SBF during resting conditions and the PRH response were observed and the dominant frequency was evaluated. The coefficient of variation for this frequency was $6 \%$ for each area. To determine whether a systematic difference existed between the first and the last test, we used Student's $t$ test. There were no significant differences between these measurements.

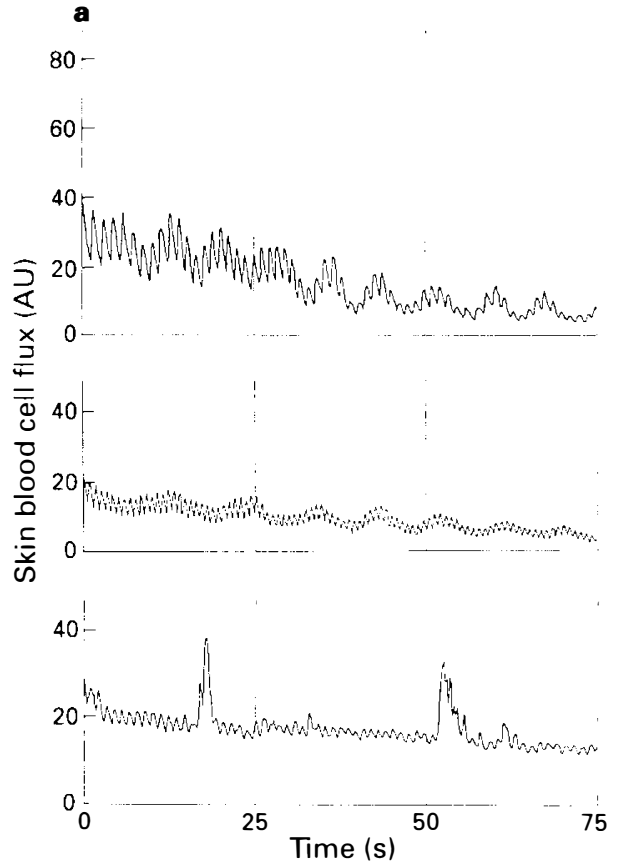

b
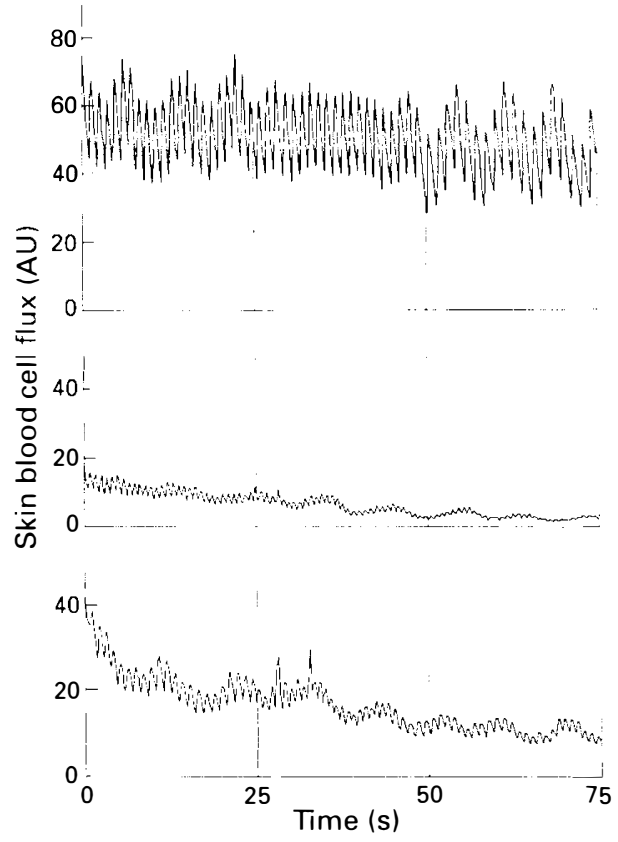

Figure 1 (a) Sacrum. Original recordings of the skin blood cell flux, as a function of time, during the post-occlusive reactive hyperaemia response in the skin over the sacrum. The recordings are from a healthy younger subject (upper), an elderly subject (middle), and a spinal cord injured subject (SCI, lower) without distinct flowmotion on the recording. In the SCI subject two large spasms occurred. (b) Gluteus muscle. Original recordings of the skin blood cell flux, as a function of time, during the post-occlusive reactive hyperaemia response in the skin over the gluteus muscle. The recordings are from a healthy younger subject (upper), an elderly subject (middle), and a spinal cord injured subject (SCI, lower). In the SCI subject two small spasms occurred. An interference pattern in the flowmotion was evident in the healthy younger subject during the time interval $25-50 \mathrm{~s}$ 

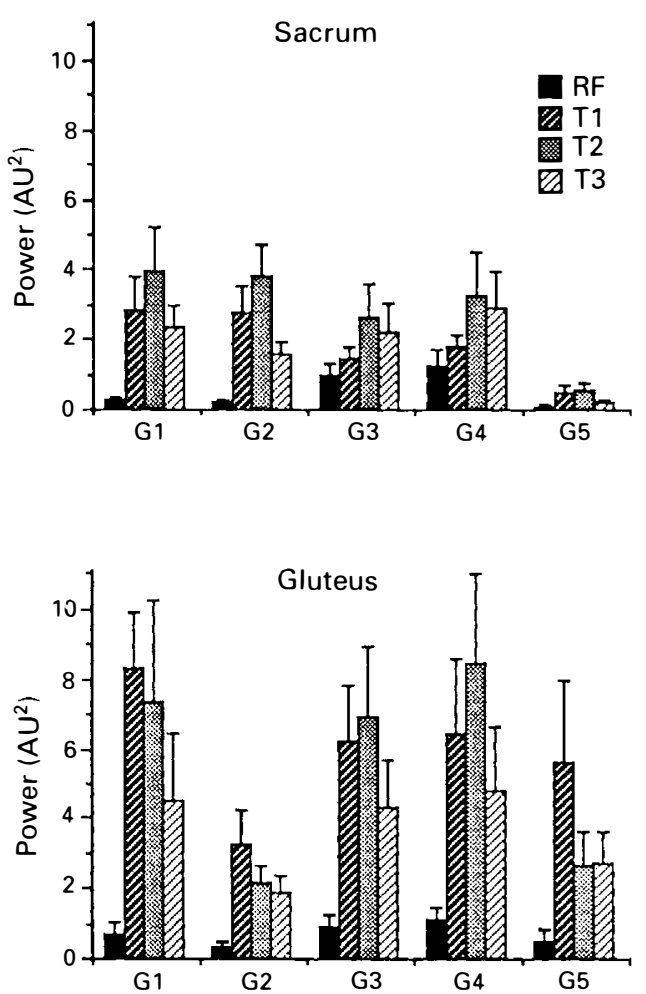

Figure 2 The power of the flowmotion (mean \pm SE) in the skin over the sacrum (upper) and the gluteus muscle (lower) during four time windows (resting conditions: $\mathrm{RF}$, and post-occlusive reactive hyperaemia: T1, T2, T3). Healthy younger subjects (G1), elderly subjects (G2), spinal cord injured subjects (SCI, G3), and two SCI subgroups (one with (G4) and one without (G5) distinct flowmotion on the original recordings) were evaluated

The study was approved by the ethical committee of the hospital, and all subjects gave their prior informed consent.

\section{Results}

The results obtained with the PSLE method have been analyzed and values of power and center frequency (mean, \pm SE, $P$ values) are shown in Table 1 and Table 2, respectively. In G2 the two categories of elderly subjects (living at home or permanently institutionalized) were analyzed separately, but since no significant difference was found the results are reported for the whole group. In G3 the analyses were made in the SCI patients grouped by level of injury (thoracic $v s$ cervical) and by presence or absence of sensation over the sacrum. No significant differences in power or center frequency were found. However, inspection of the original recordings revealed that some SCI subjects had a hardly perceivable flowmotion over the sacrum (Figure 1). Therefore, in a further analysis, the SCI subjects were divided into the following two subgroups.

Subgroup 4 (G4) comprised 15 spinal cord injured subjects with distinct flowmotion over both areas, with a mean age of 36 years (range 21-53), and a BP of
$122 / 76 \pm 3 / 2 \mathrm{~mm} \mathrm{Hg}$ with a $\mathrm{mBP}$ of $91 \pm 2 \mathrm{~mm} \mathrm{Hg}$. The spinal cord injury was at the C4-T12 level.

Subgroup 5 (G5) comprised five spinal cord injured subjects without distinct flowmotion over the sacrum area, with a mean age of 44.5 years (range 36-53), and a BP of $104 / 65 \pm 5 / 3 \mathrm{~mm} \mathrm{Hg}$ with a $\mathrm{mBP}$ of $78 \pm 3 \mathrm{~mm} \mathrm{Hg}$. The spinal cord injury was at the $\mathrm{C} 2-7$ level.

The results for the SCI subjects are reported for both the whole group (G3) and for the two subgroups (G4 and G5).

\section{Power spectrum}

The power spectra for the flowmotion over the sacrum for groups G1 and G5 are presented as three-dimensional graphs (Figure 3 ), showing the power distribution within the frequency range $0-18 \mathrm{cpm}(0-0.3 \mathrm{~Hz})$ during the four time windows (RF, T1, T2, T3). The power during the first two time windows of the PRH response (T1, T2) is spread over 2-3 separated frequency bands. During the last time window (T3) the power seems to concentrate into a single band. In several cases similar spectral patterns were seen in the other groups, and also over the gluteus muscle. Most of the power was found in the frequency bands $5.4-6.6 \mathrm{cpm}(0.09-0.11 \mathrm{~Hz})$ and $7.8-9.0 \mathrm{cpm}$ $(0.13-0.15 \mathrm{~Hz})$. During resting conditions (RF) the power was spread over several bands. The several contributing frequencies might be the cause for the interference pattern seen in the flowmotion on the original recordings (Figure 1b, upper part).

\section{The power}

The sacrum (Figure 2, Table 1)

There was no significant difference in the power during resting conditions (RF) between the healthy younger (G1), the elderly (G2), the spinal cord injured (G3) groups, and subgroup G5. However, subgroup G4 showed a significantly higher $(P<0.05)$ power compared with G1 and G5. The power during all studied PRH time windows (T1-T3) was significantly increased $(P<0.05-0.001)$ compared with its value during resting conditions for groups G1, G2, and G5. However, for the group of SCI subjects (G3) and for the subgroup G4 the power increase was significant only during time window $\mathrm{T} 2$ and $\mathrm{T} 3$ respectively.

The power during the PRH response showed a common pattern for all the groups (G1-G5), increasing from the first (T1) to a maximum in the second (T2) and then decreasing to the last (T3) time window. The power variation during the $\mathrm{PRH}$ response only reached significance for $\mathrm{G} 2(P<0.001)$ between time windows $\mathrm{T} 2$ and T3, and for the SCI groups $(P<0.05)$ between time windows $\mathrm{T} 1$ and $\mathrm{T} 2$.

The gluteus muscle (Figure 2, Table 1)

The power during resting conditions (RF) did not show any significant difference between any groups or subgroups. The power during all studied PRH time windows (T1-T3) was significantly increased 
Table 1 Mean values $\pm \mathrm{SE}$ of the power $\left(\mathrm{AU}^{2}\right)$ of the skin flowmotion over the sacrum and the gluteus muscle

\begin{tabular}{|c|c|c|c|c|c|c|c|}
\hline & $\begin{array}{c}\text { Group } 1 \\
n=10\end{array}$ & & $\begin{array}{c}\text { Group } 2 \\
n=20\end{array}$ & $\begin{array}{c}\text { Group } 3 \\
n=20\end{array}$ & $\begin{array}{c}\text { Group } 4 \\
n=15\end{array}$ & & $\begin{array}{c}\text { Group } 5 \\
n=5\end{array}$ \\
\hline \multicolumn{8}{|c|}{ Sacrum } \\
\hline \multirow[t]{2}{*}{$\mathrm{RF}$} & $0.29 \pm 0.08$ & & $0.21 \pm 0.09$ & $0.94 \pm 0.39$ & $1.25 \pm 0.50$ & * & $0.09 \pm 0.05$ \\
\hline & (2) & & (3) & & & & (1) \\
\hline \multirow[t]{4}{*}{$\mathrm{T} 1$} & $2.84 \pm 0.95$ & & $2.75 \pm 0.78$ & $1.48 \pm 0.32$ & $1.77 \pm 0.38$ & $*$ & $0.48 \pm 0.22$ \\
\hline & & & & $\uparrow$ & $\uparrow$ & & $\uparrow$ \\
\hline & & & & * & $*$ & & * \\
\hline & (3) & & (3) & (1) $\downarrow$ & $\downarrow$ & & (1) $\downarrow$ \\
\hline \multirow[t]{4}{*}{$\mathrm{T} 2$} & $3.96 \pm 1.25$ & & $3.81 \pm 0.93$ & $2.67 \pm 0.97$ & $3.28 \pm 1.20$ & & $0.53 \pm 0.26$ \\
\hline & & & $\uparrow$ & & & & \\
\hline & & & $* * *$ & & & & \\
\hline & (2) & & (3) $\downarrow$ & & (1) & & (1) \\
\hline $\mathrm{T} 3$ & $2.37 \pm 0.61$ & & $1.60 \pm 0.35$ & $2.25 \pm 0.79$ & $2.93 \pm 1.00$ & $*$ & $0.24 \pm 0.05$ \\
\hline \multicolumn{8}{|c|}{ Gluteus muscle } \\
\hline \multirow[t]{2}{*}{$\mathrm{RF}$} & $0.67 \pm 0.35$ & & $0.36 \pm 0.13$ & $0.93 \pm 0.30$ & $1.08 \pm 0.38$ & & $0.46 \pm 0.38$ \\
\hline & (3) & & (3) & (3) & $(2)$ & & (1) \\
\hline \multirow[t]{4}{*}{$\mathrm{T} 1$} & $8.30 \pm 1.65$ & $* *$ & $3.28 \pm 0.92$ & $6.21 \pm 1.66$ & $6.44 \pm 2.16$ & & $5.61 \pm 2.36$ \\
\hline & & & & & $\uparrow$ & & $\uparrow$ \\
\hline & & & & & $*$ & & * \\
\hline & $(2)$ & & (3) & (3) & (3) $\downarrow$ & & $\downarrow$ \\
\hline \multirow[t]{4}{*}{$\mathrm{T} 2$} & $7.37 \pm 2.93$ & * & $2.17 \pm 0.50$ & $6.94 \pm 1.99$ & $8.48 \pm 2.58$ & & $2.65 \pm 0.93$ \\
\hline & & & $\uparrow$ & $\uparrow$ & $\uparrow$ & & \\
\hline & & & $* *$ & $* *$ & $* * *$ & & \\
\hline & (1) & & (3) $\downarrow$ & (2) $\downarrow$ & (1) $\downarrow$ & & \\
\hline $\mathrm{T} 3$ & $4.53 \pm 1.92$ & & $1.87 \pm 0.52$ & $4.27 \pm 1.45$ & $4.78 \pm 1.91$ & & $2.73 \pm 0.89$ \\
\hline
\end{tabular}

Group 1: healthy subjects, group 2: elderly subjects, group 3: spinal cord injured subjects, group 4: spinal cord injured subjects with distinct flowmotion over the sacral area, group 5: spinal cord injured subjects without distinct flowmotion over the sacral area. Significance levels for PRH (T1, T2, T3) compared with resting conditions (RF) within a group: (1) $P<0.05$, (2) $P<0.01$, (3) $P<0.001$. Significance levels within a group during $P R H$ or between groups: ${ }^{*} P<0.05$, ${ }^{* *} P<0.01,{ }^{* * *} P<0.001$. For abbreviations, see text

$(P<0.05-0.001)$ compared with its value during resting conditions in all groups, except in subgroup G5. In this group a significant increase $(P<0.05)$ was found only during $\mathrm{T} 1$. The maximum power was found during $\mathrm{T} 1$ in $\mathrm{G} 1, \mathrm{G} 2$, and $\mathrm{G} 5$, but during $\mathrm{T} 2$ in $\mathrm{G} 3$ and $\mathrm{G} 4$. The power for $\mathrm{G} 2$ was significantly lower during $\mathrm{T} 1$ $(P<0.01)$ and T2 $(P<0.05)$ compared with $\mathrm{G} 1$.

\section{Comparison between the two skin areas}

During the PRH response, the pattern of power variation over the gluteus muscle differed from that over the sacrum, both for group G1 and G2, showing a monotonic power decrease from $\mathrm{T} 1$ to $\mathrm{T} 3$. By contrast, the SCI group (G3) showed the same power pattern over both skin areas, though the power over the gluteus muscle was significantly higher $(P<0.05-0.01)$ during $\mathrm{T} 1$ and $\mathrm{T} 2$. In subgroup G5, however, four of the five subjects showed a distinct flowmotion over the gluteus muscle in contrast to the sacrum, and the power pattern was also different compared with the sacrum area, with almost the same level during $\mathrm{T} 2$ and $\mathrm{T} 3$.

\section{Center frequency}

The sacrum (Table 2)

During resting conditions (RF) the center frequency did not show any significant differences between the groups. During the whole PRH response the center 
Table 2 Mean values \pm SE of the center frequency $(\mathrm{cpm})$ of the skin flowmotion over the sacrum and the gluteus muscle

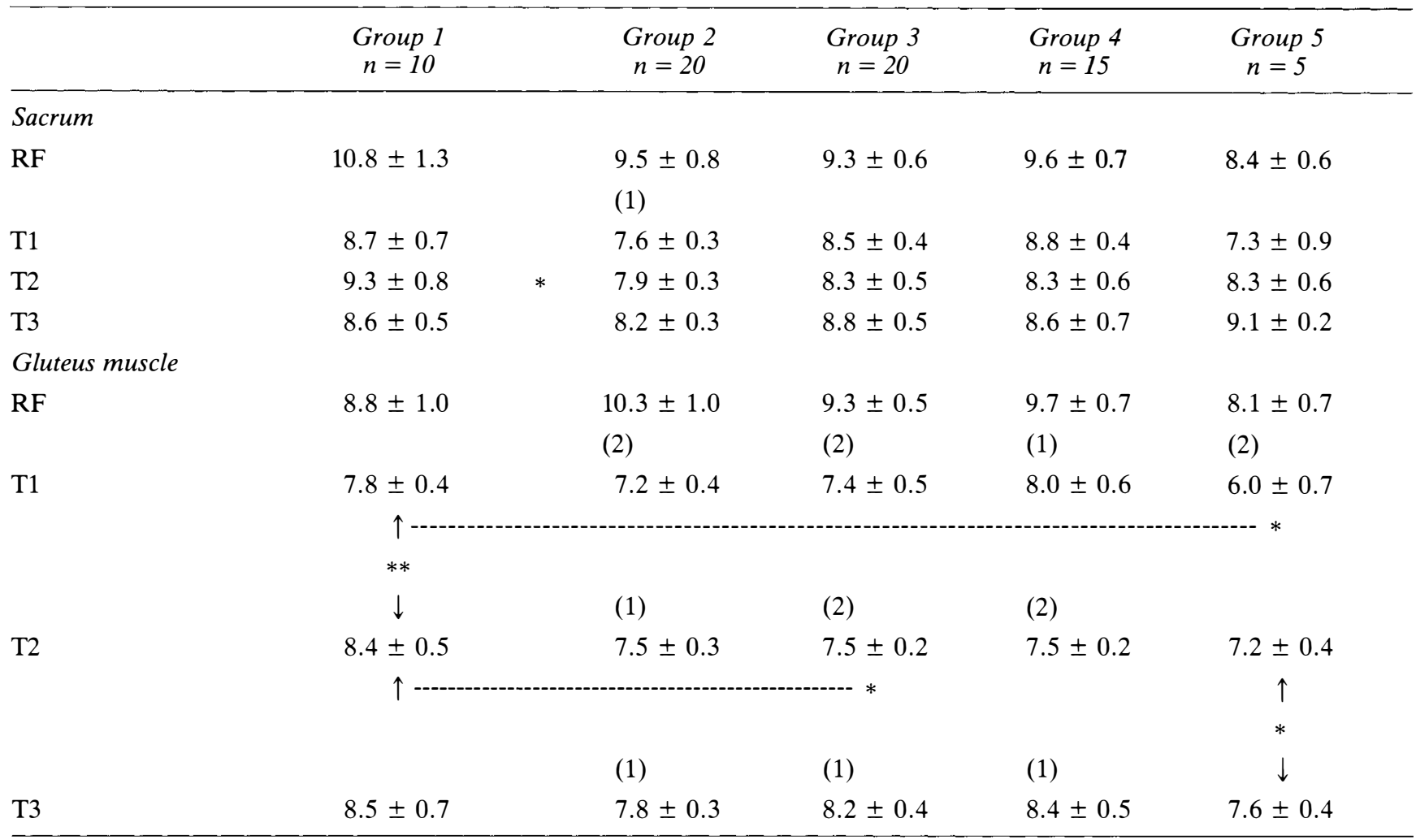

Group 1: healthy subjects, group 2: elderly subjects, group 3: spinal cord injured subjects, group 4: spinal cord injured subjects with distinct flowmotion over the sacral area, group 5: spinal cord injured subjects without distinct flowmotion over the sacral area. Significance levels for PRH (T1, T2, T3) compared with resting conditions (RF) within a group: (1) $P<0.05$, (2) $P<0.01$. Significance levels within a group during PRH or between groups: ${ }^{*} P<0.05$, ${ }^{* *} P<0.01$. For abbreviations, see text

frequency was lower compared with resting conditions for all groups, except for G5 during T3. However, this center frequency decrease was significant $(P<0.05)$ only for group $\mathrm{G} 2$ during $\mathrm{T} 1$. Between the PRH time windows (T1-T3) the center frequency did not change significantly for any group. The elderly subjects in group $\mathrm{G} 2$ showed a lower center frequency during the first two windows of the PRH compared with G1 and the difference was significant $(P<0.05)$ during T2.

The gluteus muscle (Table 2)

During resting conditions (RF) the center frequency did not show any significant differences between the groups. During the whole PRH response the center frequency was lower compared with resting conditions for all groups. This center frequency decrease was significant $(P<0.01-0.05)$ during all time windows for G2, G3, and G4. For subgroup G5 this decrease was significant $(P<0.01)$ during T1. Between the PRH time windows (T1-T3) the only significant change of the center frequency was an increase in G1 $(P<0.01)$ between $\mathrm{T} 1$ and T2, and an increase in G5 $(P<0.05)$ between T2 and T3. For subgroup G5 the center frequency during time window $\mathrm{T} 1$ was significantly lower $(P<0.05)$ than for $\mathrm{G} 1$.

\section{Comparison between the two skin areas}

Comparing the center frequencies of each time window in the sacrum and the gluteus muscle areas, it was seen that during the whole PRH response studied the center frequency for the gluteus muscle seemed to be lower in all groups. This difference was most pronounced during time window T3 in G5 $(P<0.05)$.

\section{Comparison of center frequency and dominant frequency}

During resting conditions the manually calculated dominant frequency for the sacrum area fell between $6.9-7.5 \mathrm{cpm}$ in all groups except in subgroup G5. The center frequency calculated from the PSLE data was significantly higher $(8.4-10.8 \mathrm{cpm}, P<0.05-0.01)$ than the dominant frequency in all groups, except in subgroup G5. The average center frequency during the PRH response for the sacrum area was significantly higher $(8.0-8.9 \mathrm{cpm}, P<0.05-0.01)$ than the dominant frequency $(7.0-7.5 \mathrm{cpm})$ in all groups, except in $\mathrm{G} 1$.

For the gluteus muscle the dominant frequency during resting conditions fell between 6.3 and $7.0 \mathrm{cpm}$ in all groups. The center frequency values were significantly higher $(8.1-10.3 \mathrm{cpm}, P<0.01-0.001)$ in 


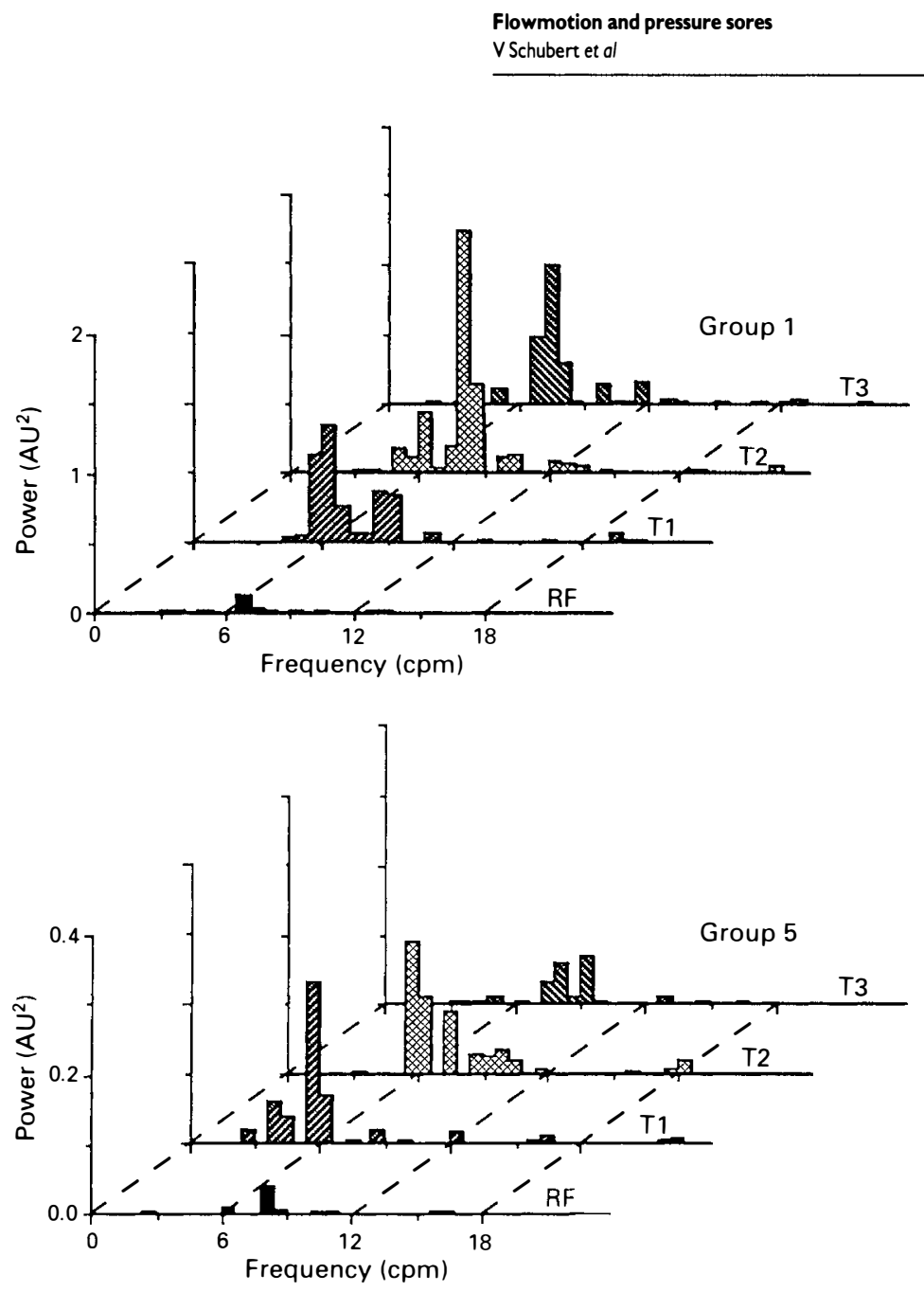

Figure 3 Power spectra for the flowmotion in the skin over the sacrum during four time windows (resting conditions: RF, and post-occlusive reactive hyperaemia: T1, T2, T3) for the healthy younger subjects (group 1, upper) and the spinal cord injured subjects without distinct flowmotion on the original recordings (group 5, lower)

groups G2, G3, and G4. For the PRH response both methods gave values within the frequency range $6.9-8.4 \mathrm{cpm}$. A significant difference $(P<0.05)$ was only found for group G3.

\section{Arterial blood pressure}

The systemic blood pressure was significantly higher in $\mathrm{G} 2(P<0.01)$ compared with $\mathrm{G} 1$. The systolic BP (sBP) in G3 was significantly lower $(P<0.05)$ compared with G1. However, the subjects in G5 showed the lowest blood pressure, both compared with G1 (sBP: $P<0.01$; dBP: NS; mBP: $P<0.01$ ) and with G4 (sBP: $P<0.001$; dBP: $P<0.01$; mP: $P<0.01$ ).

\section{Discussion}

In the present study, all SBF recordings exhibited both pulsatile oscillations related to the cardiac rhythm and rhythmic oscillations, identified as flowmotion. The latter were present over both skin areas in $90 \%$ of all cases, but were hardly perceivable over the sacrum area in $10 \%$ (subgroup G5). Del Guercio et al $^{19}$ found that vasomotion of patients with leg arteriopathies was pronounced in only $35 \%$ of the cases, slightly evident in $15 \%$, but altogether absent in the remaining cases.

The center frequency, based on the PSLE data, fell in the range of $6.9-10.8 \mathrm{cpm}$ both during resting conditions and PRH. Frequency values from human nailfold capillaries have been reported to fall in the interval $5-10 \mathrm{cpm},{ }^{20}$ and capillary flow velocity variations were found to be synchronous with the vasomotion activity of terminal arterioles in animal preparations. ${ }^{8}$

Manual evalution of the flowmotion pattern on our original recordings, except for the sacral area in G5, showed dominant frequencies in the range $6.3-8.4 \mathrm{cpm}$ for the two areas, both during resting conditions and PRH response. For the five SCI subjects in G5, the flowmotion activity was hardly perceivable on the original recordings over the sacrum (Figure 1a, lower part). However, this subgroup (G5) was also investigated with the PSLE method, and a center frequency was found within the same range as for the other groups. This information would not have been found with a manual evaluation. Comparing the center frequency with the manually evaluated dominant fre- 
quency, we noticed that the center frequencies were generally higher for all groups and areas, but within the normal range. The PSLE appears to be a more sensitive method for evaluation of flowmotion frequencies than the manual peak-counting method. In some of the SCI subjects frequent muscular spasms were registered by the laser Doppler during the investigation (Figure 1a, lower part). These spasms are known to be a potent stimulus for vasoconstriction, ${ }^{21}$ and they may contribute to difficulties in the manual evaluation of flowmotion properties.

For each group of subjects, a separate power spectrum was computed for all time windows under consideration (RF, T1-T3; Figure 3). During RF, the power was spread over a wider range of frequencies than during the $\mathrm{PRH}$ response, where the flowmotion frequencies became concentrated into two-three separated frequency bands. A possible explanation for this difference is that the recorded flowmotion activity during RF resulted from contributions by several pacemakers at separate sites, normally oscillating with different frequencies. However, the provocation by the arterial occlusion caused an increased strength of the oscillations, with a concomitant synchronization of the frequencies of neighbouring pacemakers. The few separated frequency bands might be the cause for the interference pattern in the flowmotion which was evident on direct observation of the records (Figure 1b, upper part). The activities of some local vasomotor oscillators with different frequencies might combine in an additive way during part of the time interval, creating a strong flowmotion signal. During other parts these activities might combine in a counteracting way, resulting in a weak flowmotion signal. After a while, the frequencies seemingly became more synchronized and merged into a narrow single band. This resulted in a very regular flowmotion oscillation, clearly demonstrated in G1 during the last time window (T3, Figure 3, upper part).

During the return of skin blood flow in the PRH response (T1-T3) the flowmotion amplitudes successively increased in all groups compared with their resting values (Figure 2), with a maximum power after 25-50 s for the sacrum and after $0-50 \mathrm{~s}$ for the gluteus muscle area. Thereafter the oscillations became progressively damped. Slaaf et $a l^{22}$ studied arteriolar vasomotion in the rabbit tenuissimus muscle and found that vasomotion disappeared during the first minutes of PRH. On the other hand, Wilkin ${ }^{23}$ measured post-occlusive reactive hyperaemia response of the skin on the forearm in younger subjects, and he reported that the oscillation amplitudes reached maximum $30 \mathrm{~s}$ after release of the occlusion, which coincides with the findings in the present study.

The SCI subjects in this study were at least 1 year post-injury and they had a previous anamnesis of ulceration. When the flowmotion power in the sacrum area for the SCI subgroups was compared with G1, the two subgroups showed opposite behaviours. During resting conditions G5 had a very low flowmotion strength, but G4 had a significantly higher. Previously it was found that the sympathetic outflow from the chronically decentralized spinal cord usually was weaker than when supraspinal connections are intact ${ }^{24}$ and it has also been found that may spinal lesions regarded as complete in fact are not. ${ }^{25}$ The regulation of the cutaneous blood flow is complicated and the majority of blood vessels are believed to be controlled by the autonomic nervous system which includes both sympathetic vasoconstrictor and vasodilatory inputs. A disturbed balance between these two controls may explain the difference in flowmotion power between G4 and G5, and may be a sign of disturbed autonomic nervous function in the sacrum area. Cochrane et $a l^{26}$ found that patients with neuropathic feet and severe problems with ulceration showed reduced blood cell flux and little evidence of vasomotor control. Loss of neurogenic control leads to disturbances in the flow pressure regulation which affect the skin circulation. ${ }^{27}$ In the absence of vasomotion, a reduced venular flow might lead to an increased venous resistance and thereby serve to elevate the capillary pressure ${ }^{28}$ and to a decreased outflow as well. This would cause an accumulation of metabolic waste products, and may be a contributing factor in the mechanisms leading to skin ischaemia.

Subgroup G5 showed the lowest arterial blood pressure of all groups, and the low blood pressure corresponded to very low flowmotion power. In a study of blood pressure in human nailfold capillaries, Mahler et $a l^{29}$ found that increased blood pressure was accompanied by increased blood flow, and vice versa. A low systolic blood pressure has been reported in patients with pressure sores, both in $\mathrm{SCI}^{30}$ and in elderly patients.

The flowmotion power in the elderly subjects exhibited the same pattern as in the healthy younger subjects, however, with a slightly lower power. This difference was significant over the muscle. Evaluation of flowmotion during resting conditions showed similar center frequencies for the elderly (G2), the healthy younger (G1), and the spinal cord injured (G3) subjects. During the PRH a generally lower frequency was found in $\mathrm{G} 2$ over the sacrum area compared with the other groups.

A decreased frequency of arterial flowmotion in some elderly patients has been reported ${ }^{5}$ and a diminished reactive hyperaemia response was previously found in elderly patients. ${ }^{4}$ Ageing of the skin involves loss of vasculature and a variety of dystrophic forms, characterized by increased length of the blood vessels, causing them to curve abnormally and to dilate. Dilated subpapillary vessels in the aged is usually associated with loss of papillary vessels, ${ }^{31}$ which alters the skin vascular architecture and may influence its physiological behaviour. A disturbed flowmotion activity will probably affect the distribution of blood flow within the nutritive capillary network and might contribute to low perfusion of the sacrum area.

In conclusion, the persistence of external pressure against a bony prominence is the main cause of tissue damage leading to ischaemia and occurrence of pres- 
sure sores. The risk for development of damage depends on whether the patient has changes in the skin and skin microcirculation due to the ageing process, or to disturbances in the nervous regulation from a spinal cord injury. The present study shows that

(1) Flowmotion (vasomotion) is present in the skin over the sacrum and the gluteus muscle of elderly, SCI, and healthy younger individuals, and its frequency corresponds to what has previously been reported for the activity of terminal arterioles in both animal and human studies.

(2) The flowmotion activity is increased during the initial PRH response and the vasomotor oscillations are split into several separated frequency bands, which can create interference patterns where the flowmotion signal temporarily almost vanishes. These oscillations later tend to merge into a single band.

(3) Disturbances in flowmotion power over the sacrum appeared in some SCI patients, who showed an extremely low power, but with frequencies similar to the other groups. These disturbances might indicate that the flowmotion frequency of the pre-capillary arterial microcirculation remains unaffected by denervation and is locally driven, but that the flowmotion power is centrally mediated. A weak flowmotion signal might be a sign of a disturbed nervous regulation which has a pronounced influence on capillary perfusion, causing a reduced transport from the arterial network into the venous and lymphatic systems. These disturbances might be involved in the mechanisms leading to pressure sore formation in the sacrum area.

(4) The presented new method for evaluation of flowmotion patterns makes it possible to perform statistical comparisons of two spectral properties between different groups and time windows.

\section{Acknowledgements}

Our gratitude goes to Professor L-H Zetterberg, Department of Signals, Sensors, and Systems, Royal Institute of Technology, Stockholm, for helpful discussions. This study was supported by grants from the Swedish Society of Medicine, the Foundations for Aging Research and 'Gamla tjänarinnor', and Mölnlycke Health Care. The study was also supported in part by USPHS grants HLBI 12493 and HLBI 17421.

\section{Appendix 1: quantitative mathematical description}

\section{Data preparation}

Each original SBF recording was first prepared by manually filtering out the ripple caused by the heart beats. This smoothed curve was then digitized with an ultrasonic transmitter-receiver system (Grafbar GP-7, Science Accessories Co, Southport, Connecticut), where each recording was manually traced. Since the tracing of the curve could not be performed at a constant speed, this operation gave a series of data points which were not equidistant along the curve's time axis. A new series of data points (samples: $x_{1}, x_{2}, \ldots$, $x_{n}, \ldots, x_{N}$ ), evenly spaced along the curve's time axis, was calculated using the Spline method. ${ }^{32}$

The sample points were taken with the time separation $\Delta t=0.5 \mathrm{~s}$. Thus, each $25 \mathrm{~s}$ long segment of the original recordings contained $N=50$ samples.

\section{The Prony spectral line estimation (PSLE) analysis}

The PSLE method was used to obtain a set of sinusoids characterized by frequencies, amplitudes, and phases such that their sum approximately reproduced the original recording. The approximation function is given by

$$
y(t)=\sum_{m=1}^{p} A_{m} \cos \left(2 \pi f_{m} t+\theta_{m}\right)
$$

where $p$ is the number of sinusoids. The parameters $\mathbf{A}_{\mathrm{m}}$, $\mathrm{f}_{\mathrm{m}}, \theta_{\mathrm{m}}$, and $p$ are evaluated so that the values of the approximation function at the sampling times, ie $y_{n}=y(n \Delta t)$ for $n=1,2, \ldots, N$, minimize the differences $x_{n}-y_{n}$. The method of minimization is described by Meyer et al. ${ }^{17}$ Due to the nature of the PSLE algorithm, it is desirable that the long-time average of the input data is removed. This was accomplished by applying a moving average filter to the data set and subtracting the result from the original record. The PSLE method thus gives discrete frequencies, $f_{m}$, which are not necessarily harmonically related. The highest frequency in the sum must be lower than half the sampling rate, ie less than $1 / 2 \Delta t=1.0 \mathrm{~Hz}$, in order to satisfy the Nyquist criterion. ${ }^{33}$ In order to distinguish between record segments belonging to different subjects within a group, the parameters in Eqn (1) are given an index $k$ which identifies the subject. Thus $k=1,2, \ldots, M$, where $M$ is the number of subjects in the group under study. Each flowmotion segment of an individual recording can be treated as a sample of a random process for the time window under consideration. Therefore, the parameters $A_{m_{k}}, f_{m k}, \theta_{m_{k}}$, and $p_{k}$ are random variables. A process of this type is usually described in terms of its power spectrum, ${ }^{33}$ which is defined below.

\section{Power spectrum}

First the frequencies $f_{m_{k}}$ of the sinusoids in Eqn (1) are grouped into adjacent, non-overlapping classes of equal size $\Delta f$. The classes are numbered $1,2, \ldots, j, \ldots, J$. In this study the number of classes was chosen to $J=50$ and the width of the class interval was chosen to $\Delta f=0.01 \mathrm{~Hz}$. The midpoint of the $j$ th class is defined as

$$
f_{j}=\left(j-\frac{1}{2}\right) \Delta f, \quad j=1, \ldots, J .
$$

Then, for every segment $k$, the sinusoids with frequencies $f_{m_{k}}$ within the interval

$$
(j-1) \Delta f<f_{m_{k}}<j \Delta f, \quad j=1, \ldots, J
$$

are used for computation of the power in each class $j$, defined as

$$
P_{k}\left(f_{j}\right)=\frac{1}{2} \sum_{m_{k}} A_{m_{k}}^{2}, \quad j=1, \ldots, J .
$$

This power is considered to be carried by the midpoint frequency $f_{j}$ defined in Eqn (2). Now, using the results from Eqn (4) for the group of subjects under consideration, the power for each class $j$ is computed as the mean value

$$
P\left(f_{j}\right)=\frac{1}{M} \sum_{k=1}^{M} P_{k}\left(f_{j}\right), \quad j=1, \ldots, J .
$$


Finally, the power spectrum for the group is defined as the set

$$
\left\{P\left(f_{j}\right)\right\}, \quad j=1, \ldots, J .
$$

The power spectrum is thus the collection of the powers in all the frequency classes. In order to get a simple graphical visualization of the power spectrum, a new function $P(f)$ is introduced by the identity

$$
P(f) \equiv P\left(f_{j}\right), \quad\left\{\begin{array}{l}
\text { for }(j-1) \Delta f<f<j \Delta f \\
j=1, \ldots, J .
\end{array}\right.
$$

In this the way power spectra have been presented in Figure 3 in the results section of this study.

\section{The power}

In order to make possible statistical comparisons of power spectra belonging to different time windows or groups, two characteristic features of the separate record segments are now defined.

The first characteristic feature is the power $P_{k}$ for the $k$ th segment, defined in either of the two equivalent forms as

$$
P_{k}=\left\{\begin{array}{l}
\sum_{j=1}^{J} P_{k}\left(f_{j}\right) \\
\frac{1}{2} \sum_{m_{k}=1}^{p_{k}} A_{m_{k}}^{2} .
\end{array}\right.
$$

According to Eqn (8a) it can be interpreted as the sum of the powers in all the classes, but Eqn (8b) shows that it can also be computed directly, using all the amplitudes in the Prony sum in Eqn (1). Now, using the results of Eqn (8) for the group of subjects under consideration, the power $P$ can be computed as the mean value

$$
P=\frac{1}{M} \sum_{k=1}^{M} P_{k} \text {. }
$$

Combining Eqns (5), (8a), and (9), it can be shown that the power $P$ also can be written as

$$
P=\sum_{j=1}^{J} P\left(f_{j}\right) .
$$

$P$ is thus identical to the sum of the powers in all classes of the power spectrum, and it is therefore proportional to the area under the graph $P(f)$ defined by Eqn 7 . This property of $P$ makes it possible to interpretate the power spectrum as a specification of how the power $P$ is distributed along the frequency axis.

\section{The center frequency}

The second characteristic feature is the center frequency for the $k$ th segment defined as

$$
f_{c k}=\frac{1}{P_{k}} \cdot \frac{1}{2} \sum_{m_{k}=1}^{p_{k}} f_{m k} A_{m_{k}}^{2} .
$$

It can be interpreted as the 'center of gravity'-frequency for the power $P_{k}$. Now, using the results from Eqn (11) for the group of subjects under consideration, the center frequency can be computed as the mean value

$$
f_{c}=\frac{1}{M} \sum_{k=1}^{M} f_{c k} \text {. }
$$

It is related to the 'center of gravity'-frequency of the graph $P(f)$ for the power spectrum.

\section{References}

1 Thiyagarajan C, Silver JR. Aetiology of pressure sores in patients with spinal cord injury. BMJ 1984; 289: 1487-1490.

2 Versluysen M. Pressure sores in elderly patients. The epidemiology related to hip operations. J Bone Joint Surg Br 1986; 67: $10-13$.

3 Schubert V, Fagrell B. Postocclusive reactive hyperemia and thermal response in the skin microcirculation of subjects with spinal cord injury. Scand J Rehabil Med 1991; 23: 33-40.

4 Schubert V, Fagrell B. Evaluation of the dynamic cutaneous post-ischaemic hyperaemia and thermal response in elderly subjects and in an area at risk for pressure sores. Clin Physiol 1991; 11: 169-182.

5 Schubert V, Héraud J. The effects of pressure and shear on skin microcirculation in elderly stroke patients lying in supine or semi-recumbent positions. Age Ageing 1994; 23: 405-410.

6 Schubert V, Fagrell B. Local skin pressure and its effects on skin microcirculation as evaluated by laser-Doppler fluxmetry. Clin Physiol 1989; 9: 535-545.

7 Schubert V. Hypotension as a risk factor for the development of pressure sores in elderly subjects. Age Ageing 1991; 20: $255-261$.

8 Colantuoni A, Bertuglia S, Intaglietta M. Quantitation of rhythmic diameter changes in arterial microcirculation. $A m \mathrm{~J}$ Physiol 1984; 246: H508-H517.

9 Johnson PC, Henrich HA. Metabolic and myogenic factors in local regulation of the microcirculation. Federation Proc 1975 34: 2020-2024.

10 Walmsley D, Wiles PG. Reactive hyperemia in the skin of the human foot measured by laser Doppler flowmetry: Effects of duration of ischemia and local heating. Pediatrics 1990; 74: 345-356.

11 Meyer J-U, Borgström P, Lindbom L, Intaglietta M. Vasomotion patterns in skeletal muscle arterioles during changes in arterial pressure. Microvasc Res 1988; 35: 193-203.

12 Secomb TW, Intaglietta M, Gross JF. Effects of vasomotion on microcirculatory mass transport. Prog Appl Microcirc 1989; 15: 49-61.

13 Intaglietta M, Messmer K. Microangiodynamics, peripheral vascular resistance and the normal microcirculation. Int J Microcirc Clin Exp 1983; 2: 3-10.

14 Slaaf DW, Oude Vrielink HHE, Tangelder G-J, Reneman RS. Effective diameter as a determinant of local vascular resistance in presence of vasomotion. Am J Physiol 1988; 255: $\mathrm{H} 1240-\mathrm{H} 1243$.

15 Salerud EG, Tenland T, Nilsson GE, Öberg PÅ. Rhythmical variations in human skin blood flow. Int J Microcirc Clin Exp 1983; 2: 91-102.

16 Tenland T, Salerud EG, Nilsson GE, Öberg PÅ. Spatial and temporal variations in human skin blood flow. Int $J$ Microcirc Clin Exp 1983; 2: 81-90.

17 Meyer J-U, Burkhard PM, Secomb TW, Intaglietta M. The Prony spectral line estimation (PSLE) method for the analysis of vascular oscillations. IEEE Trans Biomed Eng 1989; 36: 968-971.

18 Meyer J-U, Intaglietta M. Measurements of the dynamics of arteriolar diameter. Ann Biomed Eng 1986; 14: 109-117.

19 Del Guercio R, Leonardo G, Arpaia MR. Evaluation of postischemic hyperemia on the skin using laser Doppler velocimetry: Study on patients with claudicatio intermittens. Microvasc Res 1986; 32: 289-299.

20 Fagrell B, Intaglietta M, Östergren J. Relative hematocrit in human skin capillaries and its relation to capillary blood flow velocity. Microvasc Res 1980; 20: 327-335.

21 Cole JD, Mani R, Sedgwick EM. Cutaneous vasomotor reflexes following spinal cord injury in man. $J$ Physiol 1985; 369: 134P.

22 Slaaf DW, Tangelder G-J, Teirlinck HC, Reneman RS. Arteriolar vasomotion and arterial pressure reduction in rabbit tenuissimus muscle. Microvasc Res 1987; 33: 71-80.

23 Wilkin JK. Periodic cutaneous blood flow during postocclusive reactive hyperemia. Am J Physiol 1986; 250: H765-H768.

24 Stjernberg L. Neural and hormonal vasomotor control and temperature regulation in spinal man. Thesis, Uppsala University, Sweden, 1986. 
25 Dimitrijevic MR, Faganel J, Lehmkuhl D, Sherwood A. Motor control in man after partial or complete spinal cord injury. In: Desmedt JE, ed. Motor Control Mechanisms in Health and Disease, Advances in Neurology. Raven Press, New York, 1983 pp 915-926.

26 Cochrane $\mathrm{T}$ et al. Laser Doppler flowmetry: in the assessment of peripheral vascular disorders? A preliminary evaluation. Clin Phys Physiol Meas 1986; 7: 31-42.

27 Pyykkö I et al. Vasomotor oscillation in vibration-induced white finger. Scand J Work Environ Health 1986; 12: 389-394.

28 Zweifach BW. General principles governing the behavior of the microcirculation. Am J Med 1957; 23: 684-696.

29 Mahler F et al. Blood pressure fluctuations in human nailfold capillaries. Am J Physiol 1979; 236: H888-H893.

30 Mawson AR et al. Risk factors for early occurring pressure ulcers following spinal cord injury. Am J Phys Med Rehabil 1988; 67: 123-127.

31 Ryan TJ. Structure, pattern and shape of the blood vessels of the skin. In: Jarrett A, ed. The Physiology and Pathophysiology of the Skin. Academic Press, London, 1973, pp 577-651.

32 Schoenberg IJ. Contributions to the problem of approximation of equidistant data by analytic functions. Q Appl Math 1946; 4: part A, 45-99; part B, 112-141.

33 Milnor WR. Recording and analysis of data. In: Hemodynamics. Williams \& Wilkins: Baltimore, 1982, pp 316-333. 